\title{
Photocatalytic degradation of Malachite Green dye by modified ZnO nanomaterial
}

\author{
S MEENA, DIPTI VAYA* and B K DAS \\ Department of Applied Sciences, The Northcap University, Gurgaon 122017, India
}

MS received 5 January 2016; accepted 31 May 2016

\begin{abstract}
In this article we report a chemical sol-gel approach to synthesize zinc oxide nanomaterials capped with ethylene diamine tetra acetic acid (EDTA), citric acid and oleic acid, and to study the effect of the surface modification on their photocatalytic activity and the kinetics for the degradation of Malachite Green (MG) dye. The structural, optical and chemical features were systematically characterized by X-ray powder diffraction, scanning electron microscopy, Fourier transform infrared and UV-vis absorption spectroscopy. The objective of using the capping agents was to confine the size and control the growth and morphology of the nanomaterial. The smallest crystallite size was recorded as $29 \mathrm{~nm}$ for EDTA-capped rod-shaped $\mathrm{ZnO}$. A comparison study of the effect of the three different capping surfactants on $\mathrm{ZnO}$ nanomaterial for photocatalytic degradation of MG dye under solar light showed that EDTA with higher denticity coordinated efficiently with the surface of $\mathrm{ZnO}$ nanocrystalline catalysts and hence demonstrated better decolouration of the dye under solar light. The dye degradation followed the psuedofirst-order kinetics. EDTA proved to be the best capping agent among all the three for $\mathrm{ZnO}$ nanomaterial.
\end{abstract}

Keywords. ZnO; EDTA; citric acid; oleic acid; photocatalysis; Malchite green.

\section{Introduction}

Wastewater containing dyes emanating from textile mills and food industries is strongly coloured and carcinogenic in nature. They cause contamination to surface and ground water posing potential health hazards to animals, plant life and humans, if present above certain concentration levels. Malachite Green (MG) dye as well as its reduced form has adverse effect on reproductive and immune system [1,2]. MG is used as a food colouring additive and as a dye in silk, wool, paper, jute, cotton, leather and acrylic industries $[3,4]$. To decrease pollution load on mainstream water, it is desirable to degrade the dye into non-toxic form before its discharge. Consequently, considerable efforts have been devoted to develop a suitable purification method that can easily destroy these bio-recalcitrant organic contaminants. The type of technique employed for detoxification may also depend upon the nature of pollutant. However, heterogeneous photocatalysis employing semiconductor oxides and UV-visible light, which can convert organic pollutants into relatively innocuous end-products such as $\mathrm{CO}_{2}, \mathrm{H}_{2} \mathrm{O}$ and inorganic ions, has emerged as a promising technology [5]. Also, photocatalysis offers a good substitute to other conventional methods for water remediation, as it uses a renewable and ecologically favourable source of energy like sunlight.

The novel physical and chemical properties of nanomaterials can be exploited to enhance the photocatalytic activity processes. Owing to increased surface area as well as

\footnotetext{
*Author for correspondence (diptivaya08@gmail.com, diptivaya@ncuindia.edu)
}

changes in surface properties such as presence of more surface defects, nanomaterials function as better catalysts [6]. Also, size confinement enhances their optical properties [7]. Semiconductor metal oxide nanoparticles show size-dependent optical properties [8].

Zinc oxide $(\mathrm{ZnO})$ is one of the promising II-VI semiconductors, which has gained much attention due to its wide and direct bandgap of $3.37 \mathrm{eV}$ at $300 \mathrm{~K}$ and large exciton binding energy of $60 \mathrm{MeV}$ at room temperature [9]. $\mathrm{ZnO}$ is a highly stable, inexpensive, nontoxic and environmentally friendly semiconducting oxide material and has potential applications in diode lasers, solar cells, gas sensors, light-emitting materials and transparent UV protection films [7,9-11].

$\mathrm{ZnO}$ is highly active towards photoinduced redox reactions. It has proven to be active in the photocatalytic oxidation of different organic compounds and complete mineralization of environmental pollutants [12-23].

$\mathrm{ZnO}$ has almost the same bandgap energy as $\mathrm{TiO}_{2}$. Some studies have confirmed that it has a better efficiency than $\mathrm{TiO}_{2}$ or equal to $\mathrm{TiO}_{2}$ in photodegradation of dyes even in aqueous solutions [24]. $\mathrm{ZnO}$ can thus be used as a suitable alternative to $\mathrm{TiO}_{2}$ as a heterogeneous catalyst [25].

In order to increase the applicability of a heterogeneous catalyst, it is important to control its particle size, morphology, size distribution and dispersion. The various methods reported for synthesis of $\mathrm{ZnO}$ nanoparticles include solvothermal and hydrothermal synthesis [26-30], precipitation [31,32], polymerization method [33], laser ablation [34,35], sonochemical [36] and sol-gel [37,38] methods. The sol-gel method has gained much interest among researchers in the 
recent years as it offers controlled consolidation, shape modulation, patterning of the nanostructures and a low processing temperature $[39,40]$.

Recent literature survey shows that at very small sizes agglomeration tends to occur to reduce the surface energy. Size can be controlled and stabilized by tuning the catalyst surface by capping $[8,41]$. Also, the morphology of the synthesized particles depends upon the nature and critical concentration of the capping agents [42]. Therefore, nanomaterials functionalized with capping agents and prepared by sol-gel method can prove to be a powerful and effective route to obtain monodisperse and stable particles with narrow size distribution at room temperature.

In this study, we have used three different capping agents, namely ethylene diamine tetra acetic acid (EDTA), citric acid and oleic acid in the synthesis of stable $\mathrm{ZnO}$ nanoparticulate material of particle size approx. $50 \mathrm{~nm}$ by green chemistry approach and examined their catalytic efficacy in the photodegradation and mineralization of a model pollutant, such as MG dye in aqueous solutions. The crystal phase, particle size and microstructure were examined by (XRD), scanning electron microscopy (SEM), UV-visible and Fourier transform infrared (FT-IR) technique. Probable mechanism for photocatalytic degradation based on experimental results has also been projected.

\section{Experimental}

2.1 Preparation of EDTA-capped (ZEDTA), citric acid-capped (ZCA) and oleic acid-capped (ZOA) ZnO nanomaterial

All chemicals used were of analytical grade, purchased from $\mathrm{CDH}$ and Fischer Scientific and were used as such without further purification. Deionized water was used in all experiments as the synthesizing medium.

The preparation method for $\mathrm{ZnO}$ powder was based on the wet chemical precipitation method. Freshly prepared aqueous $(0.2 \mathrm{M}) \mathrm{Zn}(\mathrm{NO})_{3} \cdot \mathrm{H}_{2} \mathrm{O}$ was taken as precursor and aqueous $(1 \mathrm{M}) \mathrm{NaOH}$ solution was added to it slowly in drops under continuous stirring until a basic medium $(\mathrm{pH}=14)$ was achieved. $\mathrm{ZnO}$ nanocatalyst degraded crystal violet dye to a larger extent when the $\mathrm{pH}$ value of the solution was kept at 12 [43] (scheme 1). Stoichiometric ratios of solutions of different capping agents such as EDTA, citric acid and oleic acid are prepared and then mixed dropwise into the basic solution with continuous stirring. Thereafter, the reaction mixture was refluxed at $70-80^{\circ} \mathrm{C}$ for $\sim 15 \mathrm{~h}$ until a gel-like compound was formed. The gel was aged for $12 \mathrm{~h}$ and subsequently calcined at $200^{\circ} \mathrm{C}$ in air oven for 2-3 h to obtain capped $\mathrm{ZnO}$ nanomaterial. Finally, the asprepared $\mathrm{ZnO}$ nanomaterial was powdered. Ethanolic solvent system was used for synthesizing oleic acid-capped $\mathrm{ZnO}$ nanomaterial instead of aqueous solutions.

\subsection{Characterization of $\mathrm{ZnO}$ nanomaterial}

The structural characterization of the synthesized $\mathrm{ZnO}$ nanoparticulate photocatalyst materials was carried out using

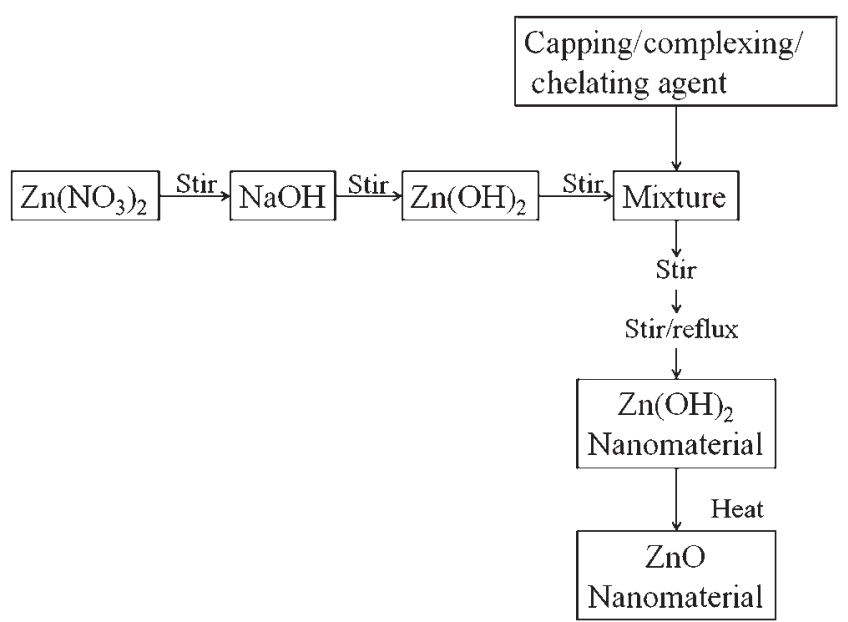

Scheme 1. Preparation of capped $\mathrm{ZnO}$ nanomaterial.

various techniques like XRD, SEM, UV and FT-IR. The crystalline phases of $\mathrm{ZnO}$ nanomaterials were identified by XRD technique. The powder XRD patterns of the $\mathrm{ZnO}$ samples were recorded using a Rigako Ultima-IV Bruker X-ray diffractometer with $\mathrm{CuK}_{\alpha}$ radiation $(\lambda=0.15418 \mathrm{~nm})$, at a $2 \theta$ scan rate of $0.5^{\circ} \mathrm{min}^{-1}$.

The surface morphology of samples was examined on a SEM Zeiss EVO 18. The absorbance spectra were recorded at room temperature using a Cary 5000 Series UV-Vis-NIR spectrophotometer both for solid samples and aqueous dispersions to find absorbance, and hence the bandgap values using Tauc's method [36]. The coordination of the capping molecules on the surface of the the nanomaterial was determind from the IR absorption spectra of materials using a Perkin Elmer Spectrum RX-1 FT-IR spectrophotometer. IR spectra was measured in the wavenumber range of 4000-600 $\mathrm{cm}^{-1}$ using $\mathrm{KBr}$ pellets and the absorption frequencies were correlated with the functional groups.

\subsection{Photocatalytic activity test on $M G$ dye}

MG (colour index no. 93405) is an organic dye having chemical formula $\mathrm{C}_{52} \mathrm{H}_{54} \mathrm{~N}_{4} \mathrm{O}_{12}$. MG is a green crystal powder with a metallic lustre, highly soluble in water and ethanol with blue green solutions.

Aqueous dye solutions $\left(1 \times 10^{-5} \mathrm{M}\right)$ of $\mathrm{MG}$ containing suspended $0.02 \mathrm{~g}$ of powdered nanocatalyst samples $\mathrm{ZP}$, ZEDTA, ZCA and ZOA were taken in separate $100 \mathrm{ml}$ beakers and simultaneously irradiated with simulated sunlight by using a 100 Watt tungsten lamp held $30 \mathrm{~cm}$ above. Thermal radiations were cutoff from the reaction mixture as they were absorbed by water contained in Petri dishes covering the beakers and the light intensity was nearly constant during the experiment. At regular intervals of $10 \mathrm{~min}, 5 \mathrm{ml}$ of aliquot samples were withdrawn from the reaction mixture and absorbance was measured at a wavelength of $610 \mathrm{~nm}$ $\left(\lambda_{\max }\right)$ using a UV-Vis spectrophotometer to follow the decolourization of MG dye. Control photolysis experiment of MG dye was also carried out simultaneously with pure 
$\mathrm{ZnO}$ catalyst (Sample ZP) under similar light exposure conditions for comparison. The decrease of absorbance values of reaction mixture at $\lambda_{\max }$ of the MG dye should be the rate of decolourization and hence photodegradation efficiency of the dye. The degradation efficiency as determined using the following relation:

$\%$ Degradation $=100 \times\left[\left(A_{0}-A_{\mathrm{t}}\right) / A_{0}\right]$,

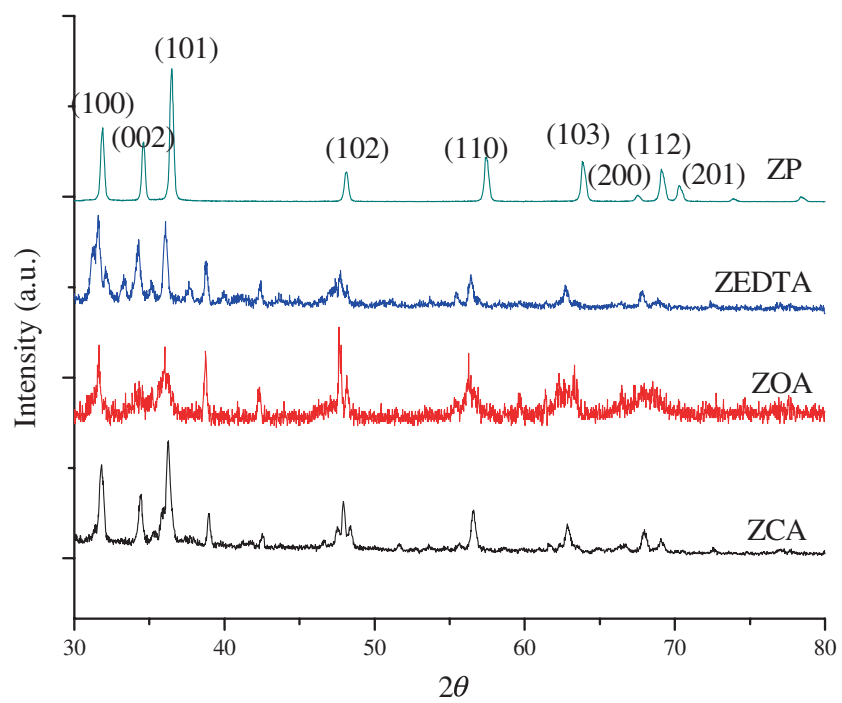

Figure 1. XRD of ZEDTA, ZCA, ZOA and ZP. XRD reflections have been indexed from JCPDS data. For ZEDTA, ZCA and ZOA peak broadening and some second phases can also be seen. where $A_{0}$ and $A_{\mathrm{t}}$ are the absorbance of the dye before and after photocatalytic reaction.

\section{Result and discussion}

\subsection{Structural characterization}

3.1a XRD analysis: From figure 1, a series of characteristic peaks: 31.55(100), 34.42(002), 36.27(101), 47.35(102), 56.37(110), 62.61(103), 66.20(200), 67.72(112), 68.87(201) were observed, which were in accordance with the Zincite phase of $\mathrm{ZnO}$ (International Center for Diffraction Data, JCPDS 36-1451). Few peaks showed the presence of second phases such as sodium citrate, sodium oleate, etc. In addition, the peaks were broad, implying particle size broadening due to very small $\mathrm{ZnO}$ particles (in nanometre range). The particle diameter $D$ was calculated using the Debye-Scherrer formula for particle size line broadening as given below:

$$
D=K \lambda / \beta \cos \theta
$$

where $K$ is Scherrer constant (i.e., 0.94), $\lambda$ the X-ray wavelength $(0.154060 \mathrm{~nm}), \beta$ the full-width at half-maximum of the (101) XRD peak and $\theta$ the Bragg diffraction angle (degree). The calculated crystallite size $D$ was $29.0 \mathrm{~nm}$ for ZEDTA, $29.11 \mathrm{~nm}$ for ZCA and $51.75 \mathrm{~nm}$ for ZOA. The so-determined crystalline sizes correlated with their photocatalytic activity (as given in section 3.2), as smallest sized ZEDTA sample has shown maximum degradation efficiency followed by ZCA then ZOA.
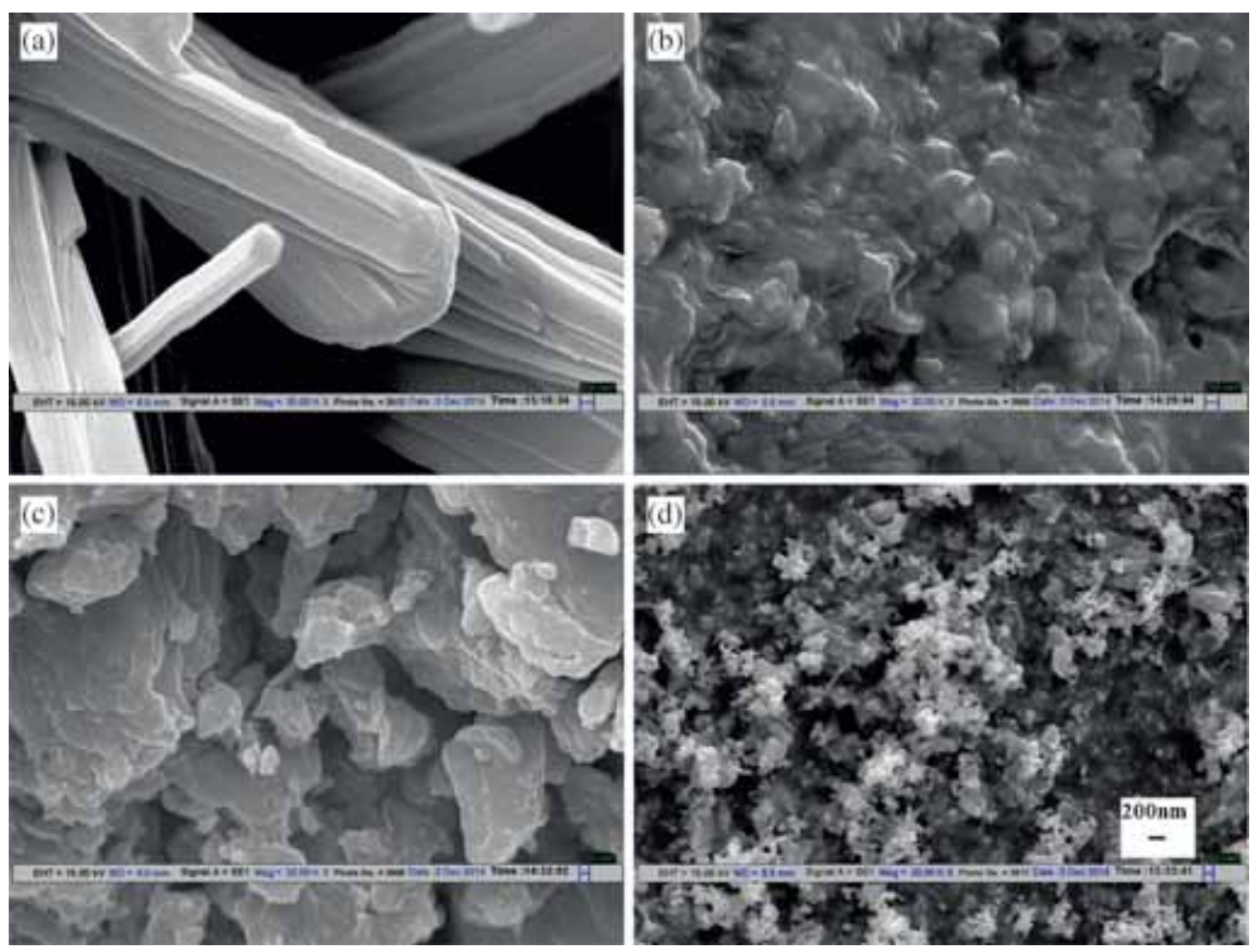

Figure 2. SEM images of (a) ZEDTA, (b) ZCA, (c) ZOA and (d) ZP. 
3.1b SEM analysis: The morphology of the as-synthesized $\mathrm{ZnO}$ powders was homogeneous, but significantly different as visible in SEM micrographs in figure 2. The interaction of the various reaction sites of the capping organic molecules with $\mathrm{Zn}^{+2}$ ion of zinc nitrate possibly changed the morphology of the resulting $\mathrm{ZnO}$ nanoparticles. The SEM micrograph of ZEDTA powder (figure 2a) showed that the powder consisted of predominantly rod-shaped structures clustered together to form bundles having width of $0.5-2 \mu \mathrm{m}$ and length in the range of $8-12 \mu \mathrm{m}$. The six reacting sites of EDTA, two nitrogen and four hydroxyl groups, can make coordination bonds with $\mathrm{Zn}^{+2}$ ion, so ZEDTA powder emerged with hexagonal morphology (table 1).

The morphology of ZCA powder (figure 2b) was almost spherical and uniform. The aggregated spheroidal particles were embedded in citric acid matrix with a broad size distri- bution up to $200 \mathrm{~nm}$. Citric acid is an excellent chelating agent with four coordinating sites and so nearly spherical shapes of nanoparticles have been formed by surface functionalization (table 1).

ZOA powder (figure 2c) showed a flaky plate-like structure made up of overlapping nanoflakes of $50 \mathrm{~nm}$ thickness and varying surface areas and distribution. Oleic acid is also an excellent capping agent that can bind strongly to the metal surface with native oxide through the single carboxyl group and ceases the growth of the particles and stabilizes them (table 1).

ZP powder (figure $2 \mathrm{~d}$ ) showed highly agglomerated particles of no definite shape.

3.1c UV-visible spectrum: The optical absorption spectra of powdered samples ZEDTA, ZCA, ZOA and ZP are shown

Table 1. Molecular structure of capping agents with number of coordinating sites.

\begin{tabular}{|c|c|c|c|}
\hline $\begin{array}{l}\text { Capping } \\
\text { agents }\end{array}$ & $\begin{array}{c}\text { Number of } \\
\text { hydroxyl groups }\end{array}$ & $\begin{array}{l}\text { Number of } \\
\text { nitrogens }\end{array}$ & $\begin{array}{l}\text { Molecular } \\
\text { structure }\end{array}$ \\
\hline
\end{tabular}

EDTA

Citric acid

Cis oleic acid
4

4

1
2<smiles>O=C(O)CN(CCN(CC(=O)O)CC(=O)O)CC(=O)O</smiles>

0<smiles>O=C(O)CC(O)(CC(=O)O)C(=O)O</smiles>

0

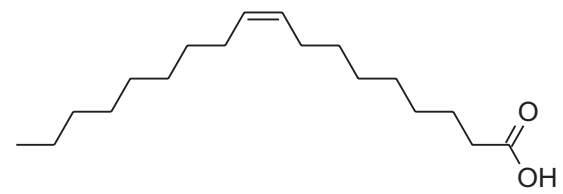

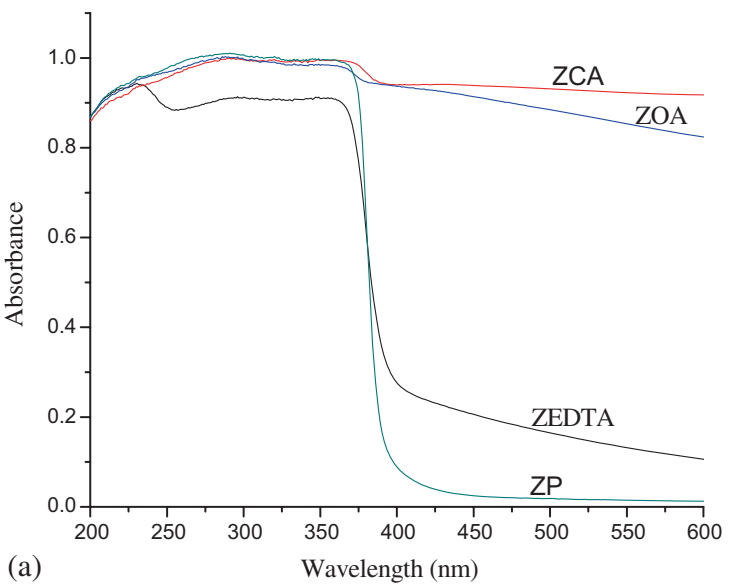

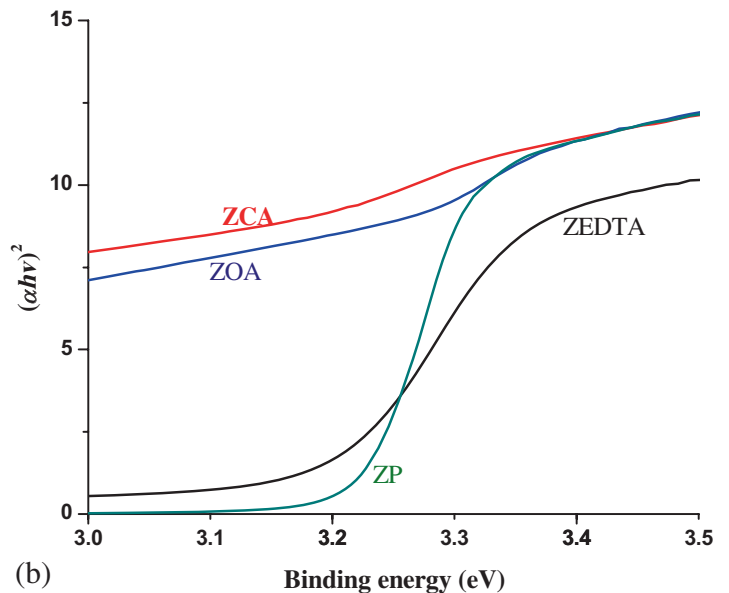

Figure 3. (a) Optical absorption spectra and (b) Tauc plot of four samples ZEDTA, ZCA, ZOA and ZP. 
in figure 3a. The effect of capping of $\mathrm{ZnO}$ on its bandgap was ascertained by their UV-visible spectra. The bandgap energies could be determined by the Tauc's method. The intercept of the tangent to the Tauc plot $(a h v)^{2} v s . h v$ (figure $3 b$ ) gives a good approximation of the bandgap energy for this direct bandgap material.
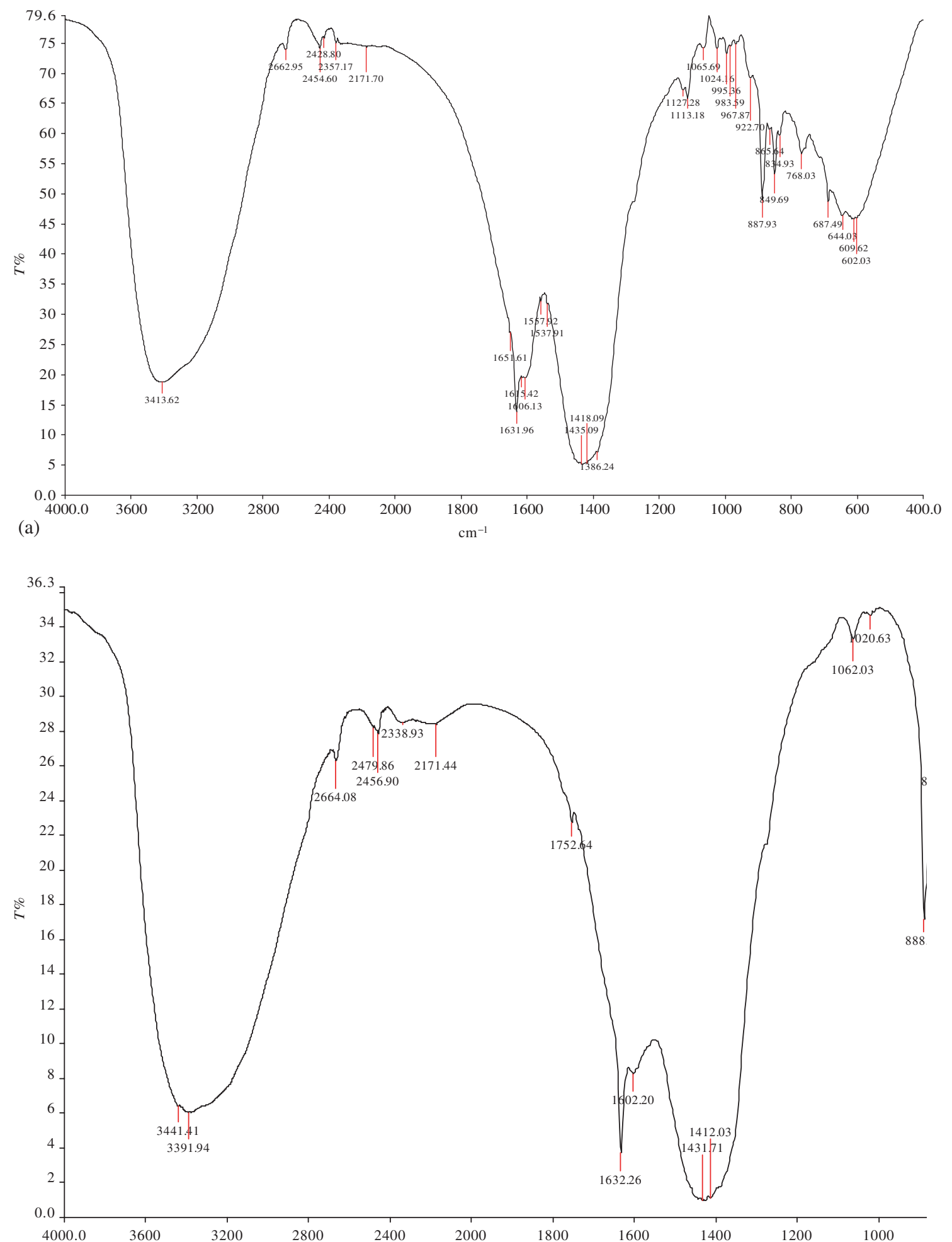

(b)

$\mathrm{cm}^{-1}$

Figure 4. IR spectra of (a) ZEDTA, (b) ZCA and (c) ZOA. 


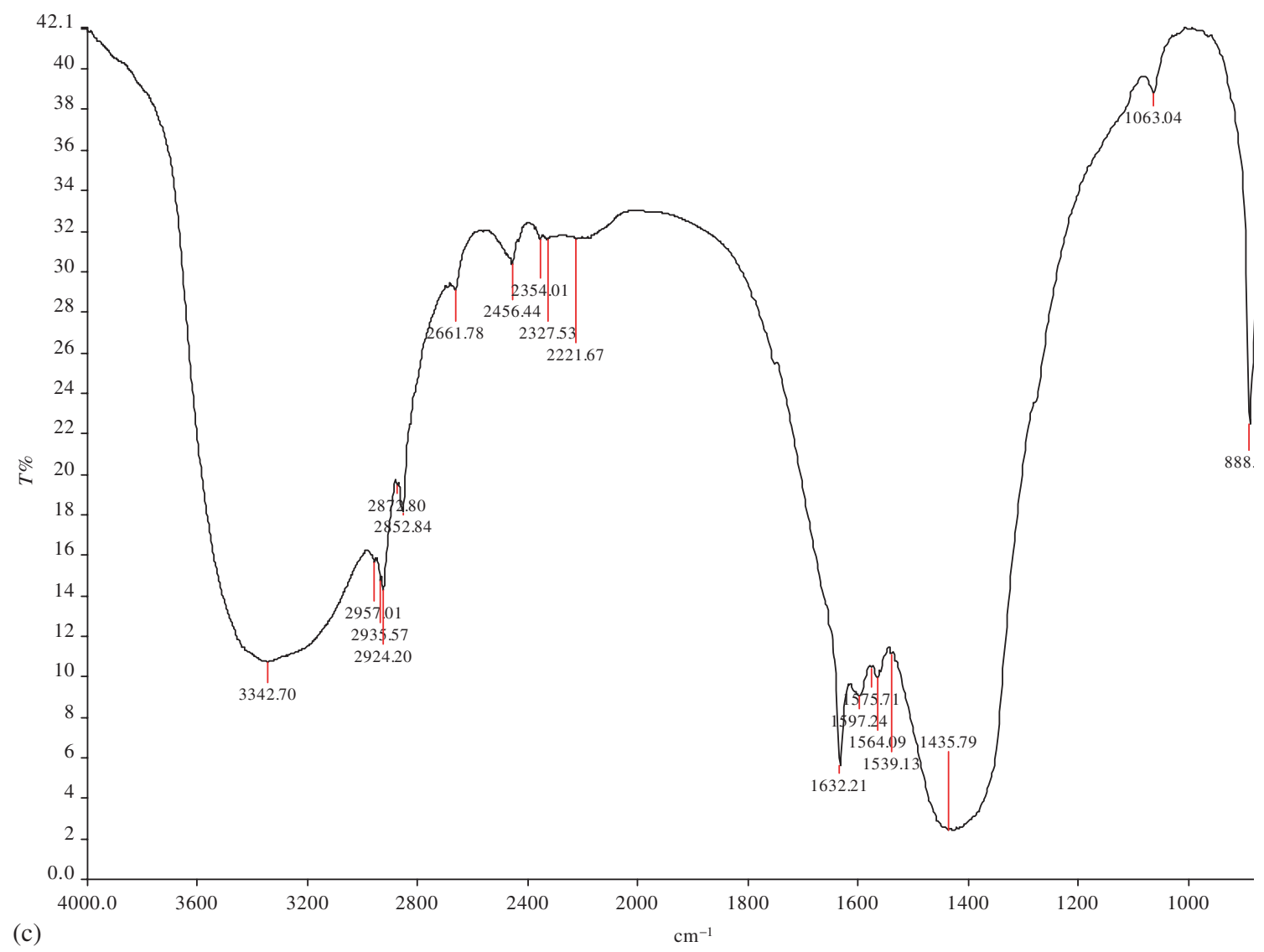

Figure 4. Continued.

Equation for direct transition using the Tauc's method is described by

$$
(a h v)^{2}=K\left(h v-E_{\mathrm{g}}\right),
$$

where $h$ is the Planck constant, $v=$ the frequency of irradiation light, $\alpha$ the absorption coefficient $\sim(1 / t) \log _{\mathrm{e}}(\mathrm{A}), E_{\mathrm{g}}$ the bandgap and $K$ the proportionality constant.

The calculated bandgap energy values were 3.15, 2.8, 2.95 and $3.2 \mathrm{eV}$ for ZEDTA, ZCA, ZOA and ZP samples, respectively. This red shift happened due to surface modification caused by complexing agents. Increased overlap of electron wavefunction leads to red shift of the absorption band [44].

3.1d FT-IR spectra analysis: In contrast with the pure IR spectra of capping agents, whose absorption bands were highly resolved, the spectra of capped $\mathrm{ZnO}$ samples EDTA, ZCA and ZOA were broader and fewer with low transmittance, clearly confirming the complexation/chelation of capping agents with $\mathrm{ZnO}$ nanoparticles.

The intense broad absorption band in the higher frequency region of all the three spectra, centered around $3400 \mathrm{~cm}^{-1}$ was due to $(\mathrm{O}-\mathrm{H})$ stretching vibrations. This was the peak of hydrogen bonded $\mathrm{O}-\mathrm{H}$ stretch in chelates, as free $\mathrm{O}-\mathrm{H}$ stretch occurs above $3600 \mathrm{~cm}^{-1}$.

The IR spectra of ZEDTA, ZCA and ZOA powders reveal absorption band structures similar to those of pure EDTA
[45], citric acid [46], oleic acid [47], respectively, except that the sharp strong absorption band at around $1700 \mathrm{~cm}^{-1}$ of $-\mathrm{C}=\mathrm{O}$ stretch from $-\mathrm{COOH}$ group shifted to a lower wavenumber of $1630 \mathrm{~cm}^{-1}$ explaining the binding of the capping agents to the surface of $\mathrm{ZnO}$ by chemisorption of carboxylate group and hence giving partial single bond character to the $-\mathrm{C}=\mathrm{O}$ group. So, we can state that the capping agents EDTA, citric acid and oleic acid have encapsulated the $\mathrm{ZnO}$ nanocrystals by binding through the carboxylate group.

On the lower frequency side of the broad band, sharp shoulders at 2925 and $2854 \mathrm{~cm}^{-1}$ corresponding to symmetric and asymmetric stretching vibrations of $-\mathrm{CH}_{2}$ groups were observed in the spectrum of ZOA (figure 4c). These arose due to the long chain of oleic acid. In ZEDTA (figure 4) and ZCA (figure 4a) due to the fewer number of alkyl groups present in them, these peaks merged with the broad $-\mathrm{O}-\mathrm{H}$ stretch and hence were not visible.

The $-\mathrm{C}-\mathrm{N}$ stretch present at $1192 \mathrm{~cm}^{-1}$ in pure EDTA shifted to $1113 \mathrm{~cm}^{-1}$ in ZEDTA (figure 4a), which indicated the coordination of EDTA to the $\mathrm{ZnO}$ surface or encapsulation of the $\mathrm{ZnO}$ crystals. The $-\mathrm{OH}$ bending peak overlapped the sharp peak at $1630 \mathrm{~cm}^{-1}$ in all the three spectra. The characteristic $-\mathrm{C}=\mathrm{C}-$ stretching peak of oleic acid also merged with this peak (figure $4 \mathrm{c}$ ). The broad intense absorption band at $1430 \mathrm{~cm}^{-1}$ could be attributed to the bending vibrations of $-\mathrm{C}-\mathrm{H}$ of methylene groups present in all the three spectra. 
The sharp peak at $888 \mathrm{~cm}^{-1}$ might be due to both $-\mathrm{C}-\mathrm{H}$ out-of-plane and $-\mathrm{O}-\mathrm{H}$ out-of-plane bending vibrations. From the above analysis complexation of capping agents with $\mathrm{ZnO}$ nanoparticle was confirmed, as chemical interaction has been established with surface of $\mathrm{ZnO}$ through the functional groups of capping agent.

\subsection{Photocatalytic properties}

The photodegradation of dye under simulated sunlight was studied by measuring the decrease in the absorbance of the dye in presence of the prepared nanopowders. The absorbance of the dye solution decreased with increase in time of exposure, indicating a decrease in the concentration of MG dye. The time-dependent degradation of MG for the samples under visible light irradiation are shown in figure 5. A plot of log absorbance $\left(A_{\mathrm{t}}\right) v s$. time (table 2) was linear and followed pseudo-first-order kinetics. The rate constant was measured with the expression given below

$$
k=\frac{1}{t} \ln \left(A_{t} / A_{0}\right) .
$$

The photonanocatalyst ZEDTA with the highest pseudo-firstorder degradation reaction rate constant was established to be more competent when compared to other nanocatalyst sample powders in photocatalytic mineralization of dyes. The efficiency of the photocatalysts, under the experimental conditions, followed the order ZEDTA $>$ ZCA $>$ ZOA $>$ ZP (table 3). After 41 min of irradiation, the maximum activity was shown by ZEDTA, ascribed likely to its heightened stability and increased surface area, as EDTA being a six toothed ligand likely served as a better chelant than the other capping molecules. There was a $380 \%$ increase in degradation constant for ZEDTA sample. This shows better results than the given literatures as indicated in table 4 .

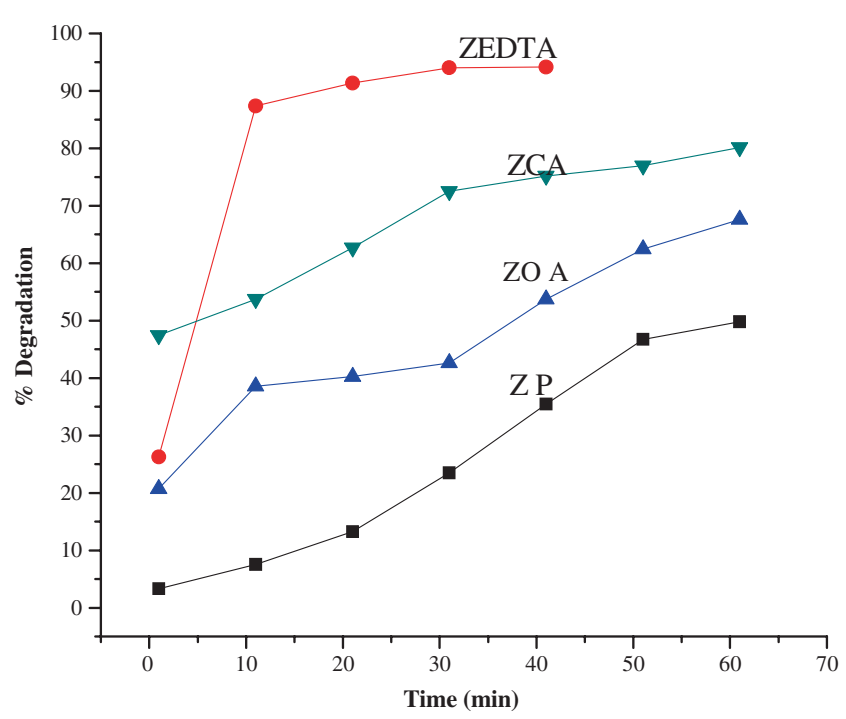

Figure 5. Percentage degradation of Malachite Green dye in the presence of various $\mathrm{ZnO}$ powders: ZEDTA, ZCA, ZOA and ZP.

\subsection{Mechanism}

On the basis of the experimental observations and existing literature, a tentative mechanism has been proposed [21] for the photocatalytic degradation of $\mathrm{MG}$ dye by $\mathrm{ZnO}$ nanomaterial capped with EDTA, citric acid and oleic acid. The photochemical-assisted formation of some reactive species is presented in the following reaction sequence.

$$
\begin{aligned}
& \mathrm{MG}_{\mathrm{ads}}^{*}+\mathrm{ZnO} \longrightarrow \mathrm{MG}^{\bullet+}+\mathrm{ZnO}_{\left(\mathrm{e}^{-}\right)} \\
& \mathrm{ZnO}_{\left(\mathrm{e}^{-}\right)}+\mathrm{O}_{2} \longrightarrow \mathrm{O}_{2}^{\bullet-} \\
& \mathrm{ZnO}_{(\mathrm{h}+)}+\mathrm{OH}^{-} \longrightarrow \mathrm{OH}^{\bullet} \\
& \mathrm{OH}^{\bullet} \text { or } \mathrm{O}_{2}^{\bullet-}+\mathrm{MG}^{\bullet+} \longrightarrow \text { degraded products } \\
& \mathrm{OH}^{\bullet} \text { or } \mathrm{O}_{2}^{\bullet-}+\mathrm{MG}_{\mathrm{ads}} \longrightarrow \text { degraded products }
\end{aligned}
$$

$\mathrm{ZnO}$ cannot absorb much of the sunlight directly due to its large bandgap $\sim 3.37 \mathrm{eV}$, but coloured organic MG dye adsorbed on its surface acts as a photosensitizer. The surface adsorbed MG was excited by absorbing solar light and donates its electrons to the conduction band of $\mathrm{ZnO}$ nanomaterials. These electrons are scavenged by dissolved oxygen to generate highly reactive superoxide oxygen radical anion $\left(\mathrm{O}_{2}^{\bullet-}\right)$. The $\mathrm{O}_{2}^{\bullet-}$ can react with surface-adsorbed $\mathrm{H}_{2} \mathrm{O}$ to form $\mathrm{H}_{2} \mathrm{O}_{2}$, which is ultimately converted to ${ }^{\bullet} \mathrm{OH}$. The surface-adsorbed $\mathrm{MG}$ radical cation or surface-adsorbed $\mathrm{MG}$ dye can undergo degradation by $\mathrm{O}_{2}^{\bullet-}$ and ${ }^{\bullet} \mathrm{OH}$ according to free radical mechanism [48]. It was confirmed that the ${ }^{\bullet} \mathrm{OH}$ radical does not participate as an active oxidizing species in the degradation of $\mathrm{MG}$, as the rate of degradation was not reduced in the presence of hydroxyl radical scavenger (2-propanol). So $\mathrm{O}_{2}^{\bullet-}$ was the main oxidizing species here.

Table 2. Absorbance of $\mathrm{MG}$ dye in the presence solar/ZnO (capped) to determine dye degradation rate constant.

\begin{tabular}{lcccc}
\hline & \multicolumn{4}{c}{$2+\log ($ absorbance) } \\
\cline { 2 - 5 } Time (min) & ZEDTA & ZCA & ZOA & ZP \\
\hline 0 & 2.003 & 2.003 & 2.003 & 2.003 \\
1 & 1.872 & 1.724 & 1.902 & 1.988 \\
11 & 1.103 & 1.672 & 1.791 & 1.969 \\
21 & 0.939 & 1.575 & 1.779 & 1.941 \\
31 & 0.778 & 1.442 & 1.761 & 1.887 \\
41 & 0.770 & 1.397 & 1.674 & 1.812 \\
51 & - & 1.365 & 1.577 & 1.729 \\
61 & - & 1.301 & 1.513 & 1.700 \\
\hline
\end{tabular}

Table 3. Rate constants and percentage degradation of MG dye by solar/ZnO (capped).

\begin{tabular}{lcc}
\hline Samples & Rate constant, $k\left(\mathrm{~min}^{-1}\right)$ & $\%$ Degradation \\
\hline ZEDTA & $58.15 \times 10^{-3}$ & 94.14 \\
ZCA & $16.95 \times 10^{-3}$ & 80.15 \\
ZOA & $13.97 \times 10^{-3}$ & 67.65 \\
ZP & $12.11 \times 10^{-3}$ & 49.40 \\
\hline
\end{tabular}


Table 4. Comparative study of pollutant degradation.

\begin{tabular}{|c|c|c|c|}
\hline Precursor, conc. of nanocatalyst & Methodology & Pollutant $\%$ degradation (timing) & Ref. no. \\
\hline Zinc acetate $(\mathrm{ZnAc}), \mathrm{NaHCO}_{3}$ & Autoclave & $\begin{array}{c}\text { Eosin red } 73 \%(60 \mathrm{~min}) \\
\text { Methyl Orange }(\mathrm{MO}) 75 \%(60 \mathrm{~min}) \\
\text { Congo red } 91 \%(60 \mathrm{~min})\end{array}$ & 14 \\
\hline $\mathrm{ZnAc}, \mathrm{EG}\left(15 \mathrm{~g} \mathrm{l}^{-1}\right)$ & Solution combustion & Rhodamine B 90\% (120 min) & 15 \\
\hline $\mathrm{ZnCl}_{2}, \mathrm{NH}_{4} \mathrm{OH}\left(1 \mathrm{~g} \mathrm{l}^{-1}\right)$ & Hydrothermal & Acridine Orange $100 \%$ (80 min) & 16 \\
\hline $\mathrm{Cu}$-doped $\mathrm{ZnO}$ thin film & Sol-gel dip coating & Methylene Blue (MB) 90\% (480 min) & 17 \\
\hline CdO-doped $\mathrm{ZnO}$ nanofibre $\left(0.5 \mathrm{~g} \mathrm{l}^{-1}\right)$ & Electrospinning & MB $100 \%(300 \mathrm{~min})$ & 18 \\
\hline $\mathrm{ZnCl}_{2}, \mathrm{NaOH}$ (sodium dodecyl sulphate) $\left(1 \mathrm{~g} \mathrm{l}^{-1}\right)$ & Wet chemical & MO $100 \%$ (140 min) & 19 \\
\hline $\mathrm{ZnAc}, \mathrm{TEA}, \mathrm{NaOH}\left(0.8 \mathrm{~g} \mathrm{l}^{-1}\right)$ & Chemical precipitation & $\begin{array}{l}\text { MO } 3.7 \% \text { (60 min); MB } 40 \% \\
\text { (60 min); MG } 62.9 \% \text { (60 min) }\end{array}$ & 20 \\
\hline $\mathrm{ZnAc}, \mathrm{LiOH}\left(0.3 \mathrm{~g}^{-1}\right)$ & Chemical precipitation & MO $70 \%(60 \mathrm{~min})$ & 21 \\
\hline $\mathrm{Zn}\left(\mathrm{SO}_{4}\right)_{2}, \mathrm{NH}_{3}, \mathrm{ZnO}$ nanoflakes & Wet chemical method & MG $76.8 \%$ (180 min) & 22 \\
\hline $\begin{array}{l}\text { Zinc nitrate, EDTA }\left(2 \mathrm{mg} \mathrm{l}^{-1}\right) \\
\text { zinc nitrate, citric acid }\left(2 \mathrm{mg}^{-1}\right) \\
\text { zinc nitrate, oleic acid }\left(2 \mathrm{mg} \mathrm{l}^{-1}\right)\end{array}$ & Sol-gel method & $\begin{array}{l}\text { MG } 94.14 \%(41 \mathrm{~min}) ; 80.15 \% \\
\quad(61 \mathrm{~min}) ; 67.65 \%(61 \mathrm{~min})\end{array}$ & This study \\
\hline
\end{tabular}

Photooxidation causes oxidation of compounds and leads to mineralization.

\section{Conclusion}

Capping agents adsorbed on specific crystalline surfaces decreased the particle growth rate, formed surface complexes and hence led to nanosizes. The various capping molecules also stabilized the nanoparticles by surface functionalization through coordination with the metal ion. The photocatalytic degradation of MG dye by $\mathrm{ZnO}$ nanomaterials as catalyst was quite effective in removal of the dye from the aqueous solution. A comparative photocatalytic activity study has been conducted for the differently capped $\mathrm{ZnO}$ nanomaterial samples. The results showed that EDTA on complexation with $\mathrm{ZnO}$ exhibited the highest photocatalytic activity. The pollutant MG degraded by $94.14 \%$ in the presence of ZEDTA, but only by $67.65 \%$ in the presence of ZOA on irradiation in the presence of visible light, probably due to the stacked nanolayer morphological structure of ZOA exposing lesser surface area for reaction. Of course, the degradation rate values were higher for all three capped samples against pure $\mathrm{ZnO}$.

Our results conclusively indicate that higher number of coordinating/chelating sites in the capping molecules most probably provide greater stability and hence show good photocatalytic ability. So, EDTA would be a suitable stabilizer for the synthesis of zincite $\mathrm{ZnO}$ nanomaterial effecting better demineralization of MG dye. EDTA molecules cap and confine the particle size to a great extent [49]. Here too, surface capping had both confining and stabilizing effect on the $\mathrm{ZnO}$ nanoparticulate material and a consequent synergistic effect on its photoactivity. The results of these studies clearly indicate that capped $\mathrm{ZnO}$ nanomaterial can catalyse degradation of dyes more efficiently.

Further studies on other capping molecules can be explored to synthesize nanoparticulate materials and their role in controlling pollution be achieved.

\section{Acknowledgements}

We thank Dr Rajender Prasad of Physics Department, Indian Institute of Technology, Delhi, for providing XRD facility.

\section{References}

[1] Rao K V 1995 Toxicol. Lett. 81107

[2] Alderman D J 1993 Clifton-Hadley R S J. Fish Dis. 16297

[3] Srivastava S, Sinha R and Roy D 2004 Aquat. Toxicol. 66319

[4] Cheng W, Wang S G, Lu L, Gong W X, Liu X W, Gao B Y and Zhang H Y 2008 Biochem. Eng. J. 39538

[5] Prado A G S and Costa L L 2009 J. Hazard. Mater. 169297

[6] Hariharan C 2006 Appl. Catal. A: Gen. 55304

[7] Meulenkamp E 1998 J. Phys. Chem. 1025566

[8] Babu K S, Reddy A R and Reddy K V 2014 Mater. Res. Bull. 49537

[9] Ozgur O, Alivov Y A, Liu C, Teke A, Reshchikov M A, Dogan S, Avrutin V, Cho S J and Morkoc H 2005 J. Appl. Phys. 981

[10] Chu S, Olmedo M, Yang Z, Kong J and Liu J 2008 Appl. Phys. Lett. 93181103

[11] Briseno A L, Holcombe T W, Boukai A I, Garnett E C, Shelton S W, Fréchet J J M and Yang P 2010 Nano Lett. 91 954

[12] Saleh $\mathrm{T}$ A, Gondal M A, Drmosh Q A, Amani Z H and Al-Yamani A 2011 Chem. Eng. J. 166407

[13] Siwen Z, Bosi Y, Yang J, Yang L, Fengyu Q and Xiang W 2014 J. Nanomater. 20141

[14] Mohammad A A, Mahmudur R I and Md E Q $2013 \mathrm{~J}$. Nanostruct. Chem. 31

[15] Sher B K, Faisal M, Mohammed M R and Aslam J 2011 Talanta 85943

[16] Jongnavakita P, Amornpitoksuka P, Suwanboonb S and Ndiege N 2012 Appl. Surf. Sci. 2588192

[17] Ayman Y, Nasser A M B, Touseef A, Afeesh R, Unnithan, Salem S A and Hak Y 2012 J. Luminescence 1321668

[18] Juan X, Yanting Li, Wei Z, Li B and Yu W 2011 Powder Technol. 207140 
[19] Lin S, Rong S, Zhidong C, Lanqin T, Yong D and Jianfei D 2012 Appl. Surf. Sci. 2585455

[20] Huihu W, Changsheng X, Wei Z, Shuizhou C, Zhihong Y and Yanghai G 2007 J. Hazard. Mater. 141645

[21] Yangang S, Yeqing Z, Yilin X, Xufeng Y, Derun D and Xijian L 2012 Micro Nano Lett. 71147

[22] Benjamin S, Vaya D, Punjabi P B and Ameta S C 2011 Arab. J. Chem. 4205

[23] Kumar G, Kumar R, Woon H S and Ahmed U 2014 J. Nanosci. Nanotechnol. 147161

[24] Dindar S and Icli J 2001 Photochem. Photobiol. A: Chem. 140263

[25] Tan T K, Khiew P S, Chiu, Radiman S, Abd-Shukor R, Huang N M and Lim H N 2011 World Acad. Sci. Eng. Technol. 5791

[26] Zhu L P, Jiao Y H, Bing N C, Wang L K, Ye L Y and Wang L J 2015 J. Nanosci. Nanotechnol. 153234

[27] Rashad M M, Ismail A A, Osama I, Ibrahim I A and Kandil A H T 2014 Arab. J. Chem. 771

[28] Razali R, Zak A K, Majid W H A and Darroudi M 2011 Ceram. Int. 373657

[29] Li Q, Kang Z, Mao B, Wang E, Wang C, Tian C and Li S 2008 Mater. Lett. 622531

[30] Xu Z Q, Deng H, Xie J, Li Y and Zu X T 2007 Appl. Surf. Sci. 253476

[31] Kuo C L, Wang C L, Ko H H, Hwang W S, Chang K M, Li W L, Huang H H, Chang Y H and Wang M C 2010 Ceram. Int. 36693

[32] Song R, Liu Y and He L 2008 Solid State Sci. 101563

[33] Jajarmi P 2009 Mater. Lett. 632646

[34] Zamiri R, Zakaria A, Ahangar H A, Darroudi M, Zak A K and Drummen G P C 2012 J. Alloy Compd. 51641
[35] Deng C, Hu Shao H and Han C 2010 Mater. Lett. 64852

[36] Khorsand Z A, Majid W H A, Wang H Z, Yousefi R, Golsheikh A M and Ren Z F 2013 Ultrason. Sonochem. 20 395

[37] Zak A K, Majid W H A, Darroudi M and Yousefi R 2011 Mater. Lett. 6570

[38] Bigdeli F and Morsali A 2010 Mater. Lett. 644

[39] Khorsand Z A, Majid W H A, Mahmoudian M R, Darroudi M and Yousefi R 2013 Adv. Powder Technol. 24618

[40] Sunandan B and Joydeep D 2009 Sci. Technol. Adv. Mater. 10 01300

[41] Lori E G, Benjamin D Y, Matt L, David Z and Peidong Y 2006 Inorg. Chem. 457535

[42] Chandrasekaran P, Viruthagiri G and Srinivasan N $2012 \mathrm{~J}$. Alloys Compd. $\mathbf{5 4 0} 89$

[43] Kaur J, Sharma M and Pandey O P 2013 J. Nanosci. Nanotechnol. 134861

[44] Donglu S, Zizheng G and Nicholas B 2014 Nanomaterial and devices, 1st edn (University of Cincinnati: Elsevier)

[45] Umamathi T, Selvi J A, Kanimozhi S A, Rajendran S and Amalraj J A 2014 Indian J. Chem. Technol. 15560

[46] Cheraghipour E, Sirus Javadpour S and Mehdizadeh A R 2012 J. Biomedical. Sci. Eng. 5715

[47] González R, García-Cerdaa L A and Quevedo-López M A 2012 Appl. Surf. Sci. 2586034

[48] Huang S T, Jiang Y R, Chou S Y, Dai Y M and Chen C C 2014 J. Mol. Catal. A: Chem. 391105

[49] Yogamalar N R, Srinivasan R and Chandra Bose A C 2009 Opt. Mater. 31570 